\title{
Publisher Correction: A molecular cross-linking approach for hybrid metal oxides
}

Dahee Jung, Liban M. A. Saleh, Zachariah J. Berkson, Maher F. El-Kady, Jee Youn Hwang, Nahla Mohamed, Alex I. Wixtrom, Ekaterina Titarenko, Yanwu Shao, Kassandra McCarthy, Jian Guo, Ignacio B. Martini, Stephan Kraemer, Evan C. Wegener, Philippe Saint-Cricq, Bastian Ruehle, Ryan R. Langeslay, Massimiliano Delferro (D), Jonathan L. Brosmer (D, Christopher H. Hendon, Marcus Gallagher-Jones (D), Jose Rodriguez (D), Karena W. Chapman (D), Jeffrey T. Miller, Xiangfeng Duan, Richard B. Kaner, Jeffrey I. Zink, Bradley F. Chmelka and Alexander M. Spokoyny (D)

Correction to: Nature Materials https://doi.org/10.1038/s41563-018-0021-9, published online 5 March 2018.

In the version of this Article originally published, Liban M. A. Saleh was incorrectly listed as Liban A. M. Saleh due to a technical error. This has now been amended in all online versions of the Article.

Published online: 16 March 2018

https://doi.org/10.1038/s41563-018-0054-0 